\title{
17 A toolkit for the intermedial analysis of computer games
}

\author{
Péter Kristóf Makai
}

This chapter is a companion to Part I's 'Media and modalities - Computer games'. In that chapter, we surveyed how intermedial theory elucidates the complex interplay of different media modalities that create a transmedial operation key to the aesthetics of the video game medium: simulation. In this chapter, we will proceed to the more practical matter of answering the question: 'how do I begin analysing video games intermedially?'

The first half of the chapter will set out a series of research steps and sample questions that intermedial scholars will want to pursue to make sense of video games through the lens of their relationship to other media. We will then provide two analyses in greater detail to show how different media, namely animation and literature enrich our understanding of video games. For simplicity's sake, we chose to include only single-player games to give prospective intermedial scholars of games a clearer idea of what a basic intermedial analysis of a game might entail. Note that although single-player games are methodologically easier to deal with, multiplayer games make up a significant portion of games played today, and they have unique aesthetic forebears. Both case studies featured here contain two parts: a general section that explores what a qualified media type (cartoons or novels) contributes to the computer game medium as a whole, followed by two case studies (of Toonstruck and 80 Days) that demonstrate how the intermedial connection is realized in a particular game. The two case studies are games with strong narrative drives, which makes them easier to compare to other narrative media, but a lot of games do not feature a story, and thus might benefit from other perspectives.

The kind of analysis performed here is not meant to be definitive or the only way of approaching the intermedial nature of games, but rather a way to showcase the capabilities of the intermedial toolbox. Don't forget: your research question might take you down a very different path. The purpose of this chapter, then, is to inspire as much as to instruct; for it is only when we make our tools do unexpected things that we can feel that we have properly learned their use.

You are welcome to consult your own scholarly traditions and research methodologies to provide new insights into the phenomena surrounding games and gaming. What follows is a set of research procedures that will help you

DOI: $10.4324 / 9781003174288-20$ 


\section{0}

Péter Kristóf Makai

investigate games using intermediality theory. This approach is geared towards the comparative view, and is most adept at highlighting the changes that occur when media are being adapted, updated, translated or remediated, or transformed in some other way.

To begin with, please note the scale of each of the phenomena to be investigated:

- A game moment (a portion or element of gameplay that is significant)

- A game mechanic (a set of repeated actions or principles of simulation in one or a number of games)

- A media product (a single stand-alone game)

- A game series (several connected titles)

- A game genre (a set of games with similar mechanics)

- The video game as a medium (a set comprising all existing and possible games)

- An intellectual property franchise (games and other media delivering a coherent set of experiences)

- Social event(s) that centre around games or gaming

- Cultural context(s) in which games are embedded

Identify the particular phenomenon, delimiting it clearly and precisely, including a description of the modalities of media that are relevant to the investigated phenomenon:

- Material: the physical interactions involved in the gaming phenomenon

- Sensory: the use of sights, sounds, tastes, smells, haptics and proprioception in the gaming phenomenon

- Spatiotemporal: the structuring of space and time in the gaming phenomenon

- Semiotic: the use of icons, indices and symbols in the construction of meanings within the gaming phenomenon

Decide which kind of intermedial relationship you are investigating:

- Media representation happens when a medium becomes the subject of a representation in a different medium. This could take many forms, such as:

- A simple representation of qualified media, e.g. game characters talking about an in-game map

- A complex representation of qualified media, e.g. film characters acting as if they were in a game

- A simple representation of a media product, e.g. a game soundtrack featuring a jingle used by a radio station

- A complex representation of a media product, e.g. a game using a soundtrack of licensed songs to evoke a particular era, genre convention or aesthetic 
- Media combination happens when more than one medium is being used to convey a particular experience or meaning in a video game. They could include:

- A simple combination of qualified media, e.g. a game using visual and audio effects to represent a character's health

- A complex combination of qualified media, e.g. a game that allows players to collect material clues, take photographs and record audio to catch a killer

- A simple combination of media products, e.g. the player playing a song in Guitar Hero (2005) by pressing buttons on the controller in sync with visual input on the screen

- A complex combination of media products, e.g. a game in which characters from different media franchises meet, such as Super Smash Bros (1999)

- Transmediation happens when the same form or content of media is mediated in a different technical medium. This can be found in any of the following:

- A simple transmediation of qualified media, e.g. the depiction of a scanned photograph in a game, or a screenshot of a game state

- A complex transmediation of qualified media, e.g. making a video recording of someone playing a game, or the film Hardcore Henry (2015)

- A simple transmediation of a media product, e.g. a cameo appearance of a film character in a game, or a shot of someone playing a particular game on film

- A complex transmediation of a media product, e.g. adapting a whole film into a game, or vice versa

- Media simulation may not be unique to the computer as a technical medium, but it is certainly the prime medium for simulations today. Media simulation happens when a target medium mimics the processes of mediation or the operations of a source medium that allows for creative mediation or the creation of novel media products. The following are some examples of this:

- A simple simulation of qualified media, e.g. a minigame in which players control a brush to produce any painting they like

- A complex simulation of qualified media, e.g. a game that simulates the workings of the film or news industry

- A simple simulation of a media product, e.g. a game mechanic that simulates hacking scenes from The Matrix (1999)

- A complex simulation of a media product, e.g. a game in which the players play as if they are the crew of a spaceship in the Star Trek universe, such as Star Trek Bridge Crew (2016) 


\section{Péter Kristóf Makai}

Compare how the phenomenon in the source medium has been modified in the target medium on the basis of the four modalities of media and bring in other points of analysis that befit the argument you are making. Your interests might lie in what gets lost during transmedial operation, in how transmedial operations reflect on the medium itself, in how each medium expands a universe of a story, in how different media are used to engage their users, or in the way transmedial media products are tied to commercial and cultural values of the day.

\section{A note on documenting observations}

While, in principle, nothing can stop a researcher from simply playing games and then relying on their general impression of how play went to make generalizations about the behaviour and the representational artefacts of the games you study, it is worth keeping in mind that the dynamic nature of computer games makes the researcher's experience ungeneralizable in many ways. Nonetheless, even if you decide that your expert knowledge is enough to investigate the intermedial phenomena in question, it is important to document your findings.

Documenting your time with the game can be as simple as taking written notes, or you may opt to record your play session in some format. These may include taking screenshots or video footage of in-game events with dedicated software. The usefulness of these observations might bring out unusual behaviours of the program, which are unique to your playthrough, so before you attribute particular significance to an occurrence, you should compare your own play sessions with other players' experiences.

Since most games challenge you directly, you might face particular obstacles that are difficult (if not impossible) to overcome in the time frame allotted for gameplay in your research schedule. To cut down on time and effort needed to get to the phenomena you wish to study, you might consider the use of paratextual aids, such as walkthroughs, strategy guides, downloading save files of characters at various stages of progress or even editing your own save files. Crowdsourced encyclopaedias of game media franchises offer helpful lexical information you can access at a moment's notice.

There is a wealth of information about how people play the games you study in various media. Recorded play sessions of other players are available in text-based, screenshot-illustrated or video-recorded formats on sites like the Let's Play! Archive, YouTube, Twitch or other websites, which might illuminate the game for researchers in new ways. However, researchers must note that these recorded sessions are also curated to particular gaming audiences, and more often than not, feature the content creator prominently, who might structure the play session towards a preconceived narrative in mind. Content creators are seldom neutral, disinterested players, and they often rely on gameplay to attain status in their gaming communities, or they might use attention-grabbing production strategies for engaging audiences to secure views and earn income from their creative endeavours. In these instances, the researcher's personal experience will be a good 
compass in determining which elements of the play session are present in the original game and which are added intentionally by the content creator for effect.

Discuss the phenomenon and situate your analysis in the relevant scholarly context or discourse:

- Disciplinary discourses, e.g. game, film, literary (or other media) studies, computer science, biology, narratology, cultural studies, communication, sociology, history, etc.

- Theoretical approaches, e.g. Marxist theory, feminist studies, critical race theory, critical disability theory, queer theory, etc.

- Methodological considerations, e.g. ethnographical studies of players, statistical methods for quantifying media reception, qualitative analysis of representations, etc.

As noted above, not all of these procedures might be equally important for every line of research. Intermedial analysis is not a checklist process in which all points should be covered in equal length and depth. The researcher is always free to omit, rephrase, modify or otherwise adapt these aspects of inquiry to suit their needs.

\section{Animation: Toonstruck}

\section{Animation in computer games}

Almost all video games are animated. Whether it is via a sparkling animation of a gemstone or the elaborate motion-captured facial performance of a real actor, the worlds of computer games come alive as a result of the painstaking work of animators. Animated entertainment has always been a heavily technical accomplishment, a home for innovation and a form of visual expression that captures the hearts and minds of its viewers. Video games are no different. This section describes how animation is created in games and shows the complex medial connections between animation that is designed for theatrical cartoons and a game that takes place in a cartoon world.

Let us begin with a brief explanation of game animation from the perspective of the technical media of display involved in the creation of moving images on the computer (Cooper 2019). All art in video games was originally 2D; since the 1980s, 3D graphics have also been available, but they only began to be widely used in the mid-1990s. Both kinds of graphical output are made from binary data. There are two major forms of organizing that data into images: raster graphics and vector graphics. In a raster image file, a (usually rectangular) bitmap defines the position and colour of each constitutive part, called pixels (from pictures, abbreviated as pix, and $e$, short for element), in a mathematical matrix. Vector graphics are created using vector paths by setting geometrical coordinates that describe lines and curves, which may then be assembled to form more complicated shapes. This way of defining shapes is more dataefficient, but they are best suited to create more abstract, geometrically 


\section{Péter Kristóf Makai}

definable images. Unlike vector graphics, raster images can be photorealistic, and individual elements can be easily manipulated.

The difference between the two ways of storing image data has implications for computer animation. Most early games and today's 2D games tend to use raster animation, which is created by displaying one bitmap after another in separate frames. In contrast, a few arcade and early PC games (such as Lunar Lander (1979) and Another World (1991)) used vector graphics because of their scalability and memory efficiency. With the advent of optical storage media, such as LaserDiscs and CDs, designers could also begin to record live-action performances, which ushered in an era of full-motion video games, bringing recognized actors to the computer screen. Notable examples include Wing Commander III: Heart of the Tiger (1993), starring Mark Hamill, John Rhys-Davies and Malcolm McDowell in lead roles, and Phantasmagoria (1995), featuring Steven W. Bailey, Jeff Rector and Douglas Seale. In full-motion video games, lifelike video footage is digitized by compressing the visual data into bitmaps - here, gameplay consists of choosing which animated clip is played as the player explores different paths of a branching narrative (one recent example is Black Mirror: Bandersnatch 2018). At the time of writing, 2020, games use 2D raster graphics or some form of 3D. In 3D, artists use vector graphics to create wire-mesh models made of polygons, on which bitmapped textures are laid and then the models are animated and rendered into the 2D image you see on the screen. The models are then made to move similarly to the principles used in stop-motion animation to capture movement, except that the computer calculates each frame in real time or slightly ahead of its display. Nonetheless, even stop-motion animation is used in games, a great example being The Neverhood (1995).

Some of the principles of hand-drawn animation are the same as those used in $2 \mathrm{D}$ animation on the computer. Backgrounds are more static and detailed than animated foreground figures in both, and are shot on separate layers. Traditional cel animation, as used in theatrical cartoons, exploits the persistence of vision to flash 24 frames per second (fps), which produces the illusion of smooth movement for the eye, but the mind can be satisfied with just $12 \mathrm{fps}$ to perceive something as animation, which is why TV animation is often 'shot on twos' (i.e. with half the frames) to cut costs. Similarly, in 2D games, backgrounds are also treated as separate from foreground objects, such as characters, whose bitmap images, called 'sprites', have livelier animations and move about independently of backgrounds. 2D platformers, for example, feature noticeable parallax scrolling, in which background layers closer to the player scroll at a quicker pace than layers further back. These similarities soon become notable in our case study, which reflects on cartoon animation as both an industry and a representative tradition.

\section{Toonstruck}

Toonstruck (1996) is a perfect example of a game where traditional animation and computer animation meet. It is also a self-reflective game in which 
animation is central to the plot and gameplay. As such, Toonstruck transmediates the animated cartoon as a medium. It mixes a filmed live-action actor playing the protagonist, Christopher Lloyd, with a hand-drawn game world, much like Who Framed Roger Rabbit? The player is put into the shoes of one Drew Blanc (Lloyd), an overworked animator at a cartoon studio known for Fluffy Fluffy Bun Bun (voiced by Tress MacNeille), which tells the story of a saccharinely cute rabbit's adventures in Cutopia. After falling asleep at the drawing board, Drew ends up within the cartoon world he helped to bring to life. He needs to wake himself up to escape from Cutopia, but to do that he must protect its residents from the evil Count Nefarious (Tim Curry). He is tasked by Cutopia's King Hugh (David Ogden Stiers) to build the Cutifier to defeat Nefarious. The simplicity of the plot and the game's visual appearance and soundscapes, as well as the logic governing the action, are exactly what we expect from Saturday morning cartoons, except that the player enacts the story, thinking of solutions to Drew's problems, rather than just sitting back and watching.

As scholars, we can use the intermedial approach to analyse several aspects of the game's use of different media in meaning-making. For example, we can highlight how Toonstruck uses cartoon animation and the conventions of the medium to provide medium-relevant clues to the logic governing the world of the computer game and to analyse the puzzle design of the game. Puzzles always leave strategic gaps in the semiotic game world, which the player must anticipate and subsequently perform. Toonstruck's intermedial connections between television cartoons and games cue players in to a very particular form of lateral thinking by transforming the cartoon world into a world in which the player has agency and prompts them to utilize intermedial forms of thinking. By using intermedial terminology, we can specify how that effect is achieved, how it informs puzzle design and how specific cues serve as elements of the design process. This, in turn, highlights the cybernetic process of give and take that takes place between machines and humans - a feedback loop that requires semiotic manipulation and intermedial operations to produce the main aesthetic effect: the cognitive challenge of the player in the service of telling a story. Thus, Toonstruck becomes more than the sum of its parts: both a cartoon and a game, it is a memorable experience and one of the top 100 adventure games ever released.

Toonstruck is a graphical adventure game, which is a submedium of the qualified media type we call the video game. This means that players who know adventure games can expect certain ways of presenting information on the screen and performing actions (hence the name 'point-and-click' adventures). Adventure games have a narrative, which the player explores by moving around in the game world, talking to characters, facing obstacles. The main challenge of adventure games is to get around those obstacles by solving brainteasing puzzles, either via finding the right items scattered around the game world and using them on other objects, or talking to characters who might provide additional bits of story or give verbal hints on how to solve the puzzle.

Materially, Toonstruck exists as computer code in the real world and exists as a program running on the technical medium of display, the home computer. It is 


\section{6}

programmed by lead programmers Douglas Hare and Gary Priest and programmers Michael Gater and Kevin Olson. Originally, Toonstruck was released on two CDs in a jewel case. Although certain data are copied onto the hard drive to make the game run, Disc One or Two needs to be in the drive for the game to be played. At the time of writing, 2020, the game is available as a downloadable programme on digital distribution platforms such as Steam or GOG, with no discs required. Because it is an old game written for DOS, the program needs to be run in an emulator, ScummVM, which is a virtual machine that mimics the behaviour of older computers.

Within the game, the cartoon logic of the world means that matter behaves rather differently from our expectations: most obviously, living people cannot inhabit a cartoon world. Christopher Lloyd's performance had to be transmediated (a bitmap sprite of his recorded actions had to be created) to make him a playable character. Drew is filmed in 3D then rendered in $2 \mathrm{D}$, and the cartoon characters are already $2 \mathrm{D}$. Thus, Toonstruck is both a complex transmediation of hand-drawn animation and a game that features a media representation of live-action performance.

Drew can easily store many, many more objects on his person than would be feasible for a real human being. This is a convention of most adventure games. And yet, in many puzzles, objects behave in a way that conforms with our expectations: mallets can hit objects, butter makes surfaces slippery, portcullises cannot be drawn up without significant force and so on. It's as if cartoon physics operates on the sole criterion of being moderately inconveniencing to the protagonist. Therefore, the game represents the physical affordances of objects and exploits them in puzzles, but it eschews simulating their full materiality for the sake of easy storage. Material interactions are brought to life with hand-drawn animation, and the possible set of interactions between objects and characters is encoded in the game itself.

As with most computer games, the players' sensory engagement with the game is visual, auditory and haptic, given that they control the game with a mouse. Therefore, the cartoon world is presented 1) in a visual format through static images (where Drew is, what he has, whom he is talking to) or animation (what he does, why he cannot perform the player's command, etc.), 2) with accompanying audio, such as background music (for ambience), spoken dialogue (for sharing the verbal knowledge of the characters) or sound effects (to highlight less visible events or to underscore interactions), while players 3) haptically communicate their intentions concerning what to do next (by moving the mouse and clicking on in-game objects).

Visually, Toonstruck is notable for being hand-drawn, animated by the Rainbow Animation and Nelvana studios and imitating the styles of Don Bluth, Chuck Jones and John Kricfalusi. The game also features animated and fullmotion video cutscenes to put game events into a narrative context without interference from the player, thus providing essential narrative beats, such as transitions between the major regions of the game. The three game worlds Drew explores, Cutopia, Zanydu and the Malevolands, feature distinct visual 
styles and colour palettes. Cutopia has a rich pastel colour scheme with a lot of curved geometry that is pleasant to look at. Zanydu has jagged, energetic lines, dynamic arrows that lead the eye and a primary RGB colour scheme with its complementing colours. The Malevolands are made up of grotesque lines and pointed ends jutting outwards; a dark palette using shades of grey, green and purple dominates.

One puzzle actively challenges the player to pay attention to colour. In Zanydu, phones do not dial numbers but colours; to call someone, a colour sequence must be pressed. In order to win a prize in a phone-in game, the player must answer questions about what colour different Zanydu objects are. However, some colours do not have designated buttons - instead, players must use the logic of additive colour theory to 'mix' the right answer.

The visual conventions of animation also feature in another puzzle: Drew at one point must obtain a star-shaped object. However, there are no persistent pickupable stars anywhere in the game. To progress, the player must recognize that hitting a cartoon character on its head with a boxing glove makes that character 'see stars'; this means that the character is shown knocked-out and a short animation plays with stars circling about their head, which then disappears. But if players are quick enough, they might notice that the cursor changes shape when they hover it over the stars, indicating that they can indeed be picked up. This requires a form of lateral thinking, since other objects are persistent and always available to the player - the puzzle exploits the representational conventions of the animated cartoon, but in this simple case of media transformation, the stars have now become interactable objects that conform to the logic of the adventure game.

One of the defining characteristics of Toonstruck is its distinctive audio profile (for sound in film, see Chapter 4). The voice acting is provided by a starstudded cast of Hollywood actors and high-profile animation voice-acting talent. The game is notable for being one of the first games to use sound effects and compositions from an actual production music library, APM, including songs such as 'Happy-Go-Lively' by Laurie Johnson, 'Workaday World' by Jack Beaver and 'Little Symphony - Spooky Scherzo' by Sam Fonteyn, as well as pieces of classical music, such as Tchaikovsky's 'Danse des petites cygnes' and Rossini's overture to La Gazza Ladra. These are examples of media representation: the game features sound effects and popular music that have previously been used by animation studios as parts of their soundtrack to strengthen the player's sense that they are in a cartoon world.

One of the puzzles is specifically audio-based. Count Nefarious's castle is protected by elaborate security technology, including voice-activated door locks. To get inside a room, Drew must modify his voice to match the squeaky tones of Nefarious's henchmen. This is achieved by obtaining a balloon from another room, quickly inhaling helium at the door and stating the name of the henchman before the effects of the helium wear off. The puzzle uses the realworld knowledge of the material behaviour of helium, and it simulates its effects by distorting the voice of Drew when the player uses the balloon on Drew. 
In terms of the spatiotemporal modality, Toonstruck the program is accessible on a PC and nowadays on Windows-compatible mobile devices, but it is not a location-based game. On average, it takes eight hours and thirty minutes to complete the game, allowing for leisurely exploration and the exhaustion of all dialogue options. In general, saving games serve an important function: it can break gaming sessions into manageable chunks of time. The freer the player is to save a game, the easier it becomes to correct one's mistakes. Earlier adventure games frequently featured death scenarios that would end the play session, requiring the player to restore an older saved game, and proceed from an earlier stage of progress. By the early 1990s, some adventure game companies have established conventions of design that avoided the player character's deaths. Toonstruck belongs to the category of games in which the player cannot be locked out of winning the game and thus having to restart due to character death or breaking the intended sequence of events to beat the game.

Toonstruck has a frame-narrative structure and is set in the real world of an animation studio, but the playable portion of the game takes place in Drew's dreams, a spatial representation of the cartoon worlds that he draws: Cutopia, Zanydu and the Malevolands. In-game, space is displayed as discrete scenes (called rooms). Rooms represent interior and exterior locations, similar to the proscenium theatre stage, with exits towards the left, right, up (or 'behind the curtains') and down (towards the audience). This is not so much a case of media representation as an instance of media simulation: the logic and the workings of black box theatre are being transmediated to form a new set of genre conventions in the adventure game.

Because Toonstruck tells a well-defined story in a chronological fashion, the story of the game progresses through 'Acts' (i.e. chapters) that determine which parts of the game world are accessible. Major storyline events are related as non-interactive cutscenes, also marking events when new areas become available. Initially, Drew arrives in Cutopia, and as the player proceeds, will eventually gain entry into Zanydu and the Malevolands. The player can travel between these three lands once they unlock the areas. The final portion of the game is set in an entirely different location, Count Nefarious' Castle, from where the player cannot access any of the other areas previously visited, thereby signalling that a showdown is at hand.

The story takes place in an undefined period of time: we know time passes, but it does not pass in an orderly fashion. Cutopia is flooded with permanent daylight, while the Malevolands are enveloped in perpetual darkness. In Zanydu, the official time is dictated by the town hall clock, which has stuck, ergo officially time does not pass. One puzzle, however, requires the manipulation of the clock: to get inside an important building, Drew needs the security guard to leave and to go home. To achieve that, Drew has to position the clock hands to $6 \mathrm{pm}$, which signals the end of the guard's shift. This puzzle works because time is only represented in the game world; it is not simulated the time of day only changes by fiat. Some obstacles, however, require timing, such as fleeing into hiding places when Nefarious's henchmen are seeking out 
Drew and Flux; here, it takes time for animations to finish, which sets the time window for our heroes to hide.

Semiotically, Toonstruck uses the above three modalities to deliver a story about the animator Drew Blanc. Players can interact with the world through a semiotic representation of the player: the in-game avatar, Drew Blanc, whom the player controls with the mouse. Because Drew is a photorealistically rendered image of Christopher Lloyd, there is an indexical relationship between Christopher Lloyd's body as recorded and its actions as Drew Blanc. Gameworld objects look similar to but not the same as real-world objects; that is, mallets are recognizably hammer-like, butter looks squishy, an ice cube looks as if it is melting. Iconic representations of inventory objects give clues to the players about their applications by referring to their affordances in the real world. However, the iconic ambiguity is resolved by a short textual description of the object at the bottom of the screen, the symbolic specificity of the words helping players to contextualize the objects. Meanwhile, characters' conversations are represented in text, symbolically, as audible dialogue performed by recognized voice actors who mimic speech impediments and give typically exaggerated vocal performances that might make it hard to pick up what is being said, especially for non-native speakers. Nonetheless, the indexical quality of the voice acting relates the voices to earlier performances in another media, which cements Toonstruck's connection to the Saturday morning cartoon.

Some objects are shown to be interactable (when the player moves the mouse cursor over them, an animation plays, and the object's name appears in text at the bottom of the screen) while others are not (as noted above, this aspect is a helpful clue when trying to solve the 'stars' puzzle). When the player engages in talking, conversation options show up at the bottom of the screen as icons in coloured bubbles: the default option for small talk, for example, is an ice cube that melts as the characters go through their available dialogue. It is the literalization of a metaphor, thereby showing both an iconic and a symbolic component to conversations. Drew also has a companion character, a toon called Flux, who talks and walks, but some puzzles require the player to use Flux as an object (e.g. as a bowling ball to score a strike). This shift between the two categories of character and object prompts players to shift their view of the materiality of everything in the game.

Most puzzles require the player to pay attention to what is happening on the screen and what characters say, as their dialogue might include hints on how to solve a particular puzzle. An example of this is that one of the things Drew needs to build the Cutifier is a cloak, and the only person who has one is the Carecrow (he is a scarecrow acting stereotypically campy, hence the name). But he will part with his cloak only if Drew can find him the costume of his dreams. Finding the right costume requires browsing through the costume shop's selection. The Carecrow says:

I want something I can wear all day long, and all year 'round - but it doesn't have to be out of this world. I don't want to have a hair out of 
place. Nothing too sappy, and not too many bells or whistles. I don't want a big top - and definitely no cross-dressing.

To solve the puzzle, the player must interpret these desires in terms of which costumes the Carecrow's needs exclude from the costume shop's catalogue, shown as pictures. Here, the player must translate the verbal descriptions into visual terms, giving a cross-modal solution to the puzzle. Of the 12 costumes, the pyjamas, the tutu, the Easter Bunny and the Santa costume, the astronaut suit, the hunter and Viking outfits and the jester, the policeman, the pope and the clown costumes all have iconic attributes that exclude them, which leaves Drew with the only correct solution: the harlequin costume - a very fitting outfit for a Carecrow.

\section{Summary: Toonstruck}

This section intended to demonstrate that video games in general, and graphical adventure games in particular, use animation to represent events of their storyworlds on the monitor, and as such, they rely on the principles of animation originally devised for the silver screen and the television set, along with new technological solutions afforded by the computer game medium. The case study of Toonstruck highlights how the audiovisual presentation of the game evokes the Saturday morning cartoons throughout the game world, how they inform game design, and how the genre of the graphical adventure game conventionalizes the player's interaction with the game world. Analysis of the game using the four modalities underscores that the choices of the game designers facilitate immersion into the game world, and also cue in the player to use the logic of the animated cartoon to solve certain puzzles. Together, they reinforce Burst Studios' creative effort to bring the experience of a human inhabiting a cartoon world to the player, an old premise of animation that originates from the earliest of Disney shorts, and live on to this day.

\section{Playable literature: 80 Days}

\section{Computer games and literature}

Casting our historical net wide, we can discover many coin-operated antecedents to the pleasures of digital gaming (Kocurek 2015). Even prior to computers dedicated to video gaming, oscilloscopes and mainframe computers were being used to display purely visual games, like William Higginbotham's 1958 program, Tennis for Two, or Steve Russell's 1962 game, Spacewar!. But a curious tradition of games emerged in the early days of computing, which did not use graphics at all: they were purely textual. There are several names for these programs, each highlighting a different aspect of mediality. The initial term in the industry was simply 'adventure game', named not for its fictional content but to signal that the game was 'like Adventure', the earliest of its type. 
Later to be called a text adventure to signal its difference from newer graphical games like Toonstruck, Adventure was written by Will Crowther and amended by Don Woods between 1975 and 1976 (Crowther and Woods 1975-6). It was an interactive exploration of a cave that was filled with fantastic beasts and magic and was influenced by the creation of Dungeons and Dragons (1974), the first role-playing game of fame (Barton and Stacks 2019, 34-8). The cave featured in the game is a faithful textual replica of the Bedquilt region of the Mammoth Caverns in Kentucky, which Crowther, an avid caver, helped survey (Jerz 2007).

A textual adventure consists of snippets of texts displayed on the computer screen, which constitute parts of a narrative. Because these snippets tell a coherent story with vivid, literary descriptions of scenes and events, and since the player is constantly prompted to type in what they want to do in the game world, this type of game is also called interactive fiction (Montfort 2003). Text adventures all feature a system for processing natural language, called a parser, which interprets player input that is phrased as two-to-four-word imperative structures, such as GO NORTH or USE MATCH ON LAMP. Every text input invites a response from the machine, and if the correct input is used, the story progresses with another snippet of text. Parsers usually have a limited vocabulary that treats synonyms differently, meaning players will have to correctly intuit the actual command needed to progress. The goal is to explore and navigate the space by removing obstacles and to reach the final room (such as an exit from a maze).

Some works of interactive fiction feature such finely crafted language, clever riddles and emotional storylines that they can rightfully aspire to the lofty title of electronic literature (Hayles 2008). They can also be called hypertext fiction (Ensslin 2007), because the snippets of text are meant to be read in a non-linear fashion, with some words forming hyperlinks to other snippets. Finally, some video game scholarship refers to text adventures as ergodic literature, coined by Espen Aarseth (1997) from the Greek words ergon (work) and hodos (path), suggesting that it takes effort from the players to read the work by carving out a path in the text that matches the order in which they read the snippets. As pieces of fiction or literature, many of the tools of literary theory can be fruitfully applied to investigate text adventures. Therefore it should not come as a surprise that literary theorists were among the first game studies scholars.

On a more general level, text is central to conveying information in games. Even in titles where text is secondary to the audiovisual and interactive elements or kept to a minimum, text is used in game menus to configure the software to the player's taste. Text often shows up on user interfaces as a verbal representation of the player's commands, as button names or as tooltips that disambiguate the meaning of otherwise purely visual icons. Spoken dialogue can be represented in text, and for many games, this might be the only medium available to represent speech, since employing voice actors is expensive. Last but not least, in-game objects can also be textual, such as signs, notes, letters or books. Some gamers object to lengthy textual descriptions, especially 
in more action-oriented gameplay, because reading text does stop the flow of events, forcing a spell of passivity on the player. Nonetheless, the right amount of text facilitates gameplay by giving the player relevant cues about what action to perform and how the story is developing and also provides verbal feedback on the success and failure of actions taken; in addition, it mediates sensory information that would otherwise be impossible to convey (such as smell, touch, pain and heat perception).

The choice of font can matter a great deal. Distinctive fonts used in game menus and logos can create new visual meanings: Sprint 2 says 'retro gaming' like no other font, The Price Is Right's PriceDown font today is more readily associated with the Grand Theft Auto universe, the use of Morpheus and Friz Quadrata now immediately evoke the UI of World of Warcraft (2004), while Caslon Antique is the font of choice for many games set in the Renaissance and the early modern periods. This just goes to show that written language is not only a form of symbolic signification but also has important iconic aspects: how it is typeset can convey mood and attitude and can evoke distinct eras. Fonts are so characteristic that they signify actual characters in the game Undertale (2015): the character Papyrus uses the eponymous font for dialogue, whereas Sans, Papyrus' brother, speaks in the font Comic Sans, and provides comic relief.

Games often play with text and textuality, even when they are graphical, further underscoring their iconic functions. Players have to quickly type in words as they appear on the screen in The Typing of the Dead: Overkill (2013) to dispatch zombies. Alan Wake (2010) has a dream sequence in which the protagonist, a writer of horror fiction, must shine his torch on words that appear in the game world to transform them into actual objects - a shift from symbolic to iconic representation is brought on by the player. Similarly, in Scribblenauts Unlimited (2012), players can create any object by typing it in, and it then becomes part of the game world. Finally, Type: Rider (2013) is literally a game about typography, in which the player controls a colon (the punctuation mark) and must jump from platform to platform in a world constructed by characters and book-related objects, taking the player through the history of typography. These are just some of the many creative uses of textuality in games, but perhaps they give you a good idea of how textuality can be transmediated and made into integral elements of gameplay.

\section{Days}

One shining example of how computer games adapt the conventions of literature is the textual adventure game 80 Days (2014). 80 Days is a postcolonial rewriting of Jules Verne's (1872) Le Tour du monde en quatre-vingts jours. Set in an alternative historical vision of 1872, the game adopts the genre conventions of steampunk fiction (VanderMeer and VanderMeer 2008): steam machines are the primary source of energy, technological inventions take centre stage, automatons exist, airships are a major form of transportation and ideas important to Victorian society are re-examined under the critical lens of the twenty-first- 
century political imaginary. As such, 80 Days is both a complex transmediation of Jules Verne's fictional universe and a re-righting (see Chapter 13 for further discussion) of the colonialist assumptions of imperial adventure.

The player is placed in the role of Passepartout, who is hired by Phileas Fogg to help him win his famous wager at the Reform Club. Passepartout has full control over where and how the duo travels. Instead of re-enacting the novel's itinerary of London-Suez-Bombay-Calcutta-Hong Kong-Yokohama-San Francisco-New York-London (which would be a case of media representation), the player can visit over 150 cities across the globe (which makes this more of a media simulation). Passepartout also has control over what they take with them: objects can be bought and sold in the cities, which might include maps, gifts or valuable artefacts. A basic economic system is implemented in the game, providing a gameplay challenge: travel is expensive and the player's funds are limited, so to pay for tickets, Passepartout must buy artefacts at low prices and sell them at high ones as they travel. Time is also an important constraint: although the game doesn't end if the duo does not reach the Reform Club in 80 days, it does result in Fogg losing his wager.

80 Days is an exquisitely crafted piece of playable literature. At a total of 750,000 words, if it could be read in its entirety, it would be longer than War and Peace, the Lord of the Rings or the first five books of the Harry Potter series. However, on any given playthrough, only a fraction of the text will be ever read by the player; the part that relates to the journeys they take and the cities they actually visit. Because the game is a piece of interactive fiction, the player makes choices about what the characters will do or say by highlighting and clicking on one of several options presented in a text box. The game's text is cut up into small snippets of text. Game studies scholar Espen Aarseth (1997, pp. 75, 119, 154) calls these textons, discrete pieces of text as they are stored in the game files; then, during play, these textons appear on the screen according to the display logic of the game, which Aarseth calls a scripton. This highlights the fact that there are important differences between what is encoded in the game and what players actualize during gameplay. There is an ambiguity in Aarseth's formulation, however: his terms may also be interpreted to distinguish between textons as single units of texts and scriptons as one particular combination of scriptons in chronological order as experienced by the player. To elucidate this latter concept: in 80 Days, a circumnavigation usually touches $20-5$ cities of the 150 -odd ones available, and every city has a handful of scenarios and events that may occur, some of which are mutually exclusive. As such, the possible scriptons are several orders of magnitude higher than the textons.

The style of the narration encountered in the game mimics the prose of Victorian authors; it is full of choice archaisms and period-appropriate slang language in its finest livery. Passepartout peppers his story with French colloquialisms, and while both he and his master speak certain foreign languages, they are almost never represented in text. As an adaptation, it takes significant liberties with its source material, but there are plenty of references to other literary works: in Nassau, the player can attempt to travel to the moon at the 


\section{4}

insistence of Michel Ardan, the French adventurer from Verne's De la terre à la lune (Verne 1865), they can embark on a journey to the North Pole with assistance from Otto Lidenbrock, the German professor from Voyage au centre de la terre (Verne 1864), and they may rub shoulders with Captain Nemo from Vingt mille lieues sous les mers (Verne 1869-70) aboard the Nautilus if they opt to travel from Bhayi to Saint-Denis. These would be examples of complex media representations, since they are not only present in the story, but Passepartout can have conversations with the characters; these are original dialogues. Fogg and Passepartout can even meet Jules Verne himself aboard the Amphitrite Express, a submersible train that crosses the English Channel underwater. These efforts to incorporate the whole oeuvre of Jules Verne is a result of the radical opening up of the storyworld, giving more options to the player but also constituting a gesture towards creating a transfictional world that connects characters in the same way hypertexts link together separate texts (for more on transmedial worlds, see Chapter 13).

In terms of the material modality, 80 Days is presented as a computer program with a unique graphical interface that enables the manipulation of an in-game map and the contents of Passepartout's coffers and the text to be displayed using light waves and represented auditorily using sound waves to deliver an atmospheric score. The originally intended technical medium of display was the iOS 7, which entailed making a series of design choices to suit touchscreens, such as having a sparse user interface, specific fonts for easy readability, a font size appropriate for touching, and a simple but striking visual style. Later, the game was ported for Android, Windows, Mac OS X and the Nintendo Switch. Its code was written in 'ink', a mark-up language of the developers' own devising that supports branching narrative creation and can be integrated into the Unity engine. The use of ink allows the story of 80 Days to branch out into different event sequences based on the player's previous choices and conditions that the game tracks. Because it is just code, the story is also much easier to expand than a printed novel, whose text seldom changes as radically as a game narrative's. A major update of the Windows edition introduced over thirty new cities and several new storylines (some 250,000 additional words) to users old and new, challenging players to explore them. This just goes to show that a scholar cannot assume that a game will remain the same after he or she has conducted their analysis.

In the sensory modality, the game communicates with the player visually through the use of icons and images, verbally in text, that is, in menus, the user interface and the main text box where the narrative is displayed, and auditively via the game's sound effects and soundtrack (no dialogue is recorded), whereas the player gives haptic feedback via the touchscreen.

Visually, the art style of the game is, anachronistically, art deco, which communicates the idea to the player that the game world is not going to be faithful to the period either; instead, it evokes a more retro-futuristic atmosphere. The style was chosen by Jaume Illustration for more practical reasons as well: the iOS 7 update changed how user interfaces appeared, so the flat look and the use 
of primary colour highlights were a natural fit for the new environment. Despite the geometric look, the graphics were not created in a vector editor, but produced as bitmaps. The eye-pleasing pastel colour scheme changes as the globe spins and day turns into night, while vehicles are shown as black-and-white silhouettes to make them stand out, but leave the details to the imagination.

In terms of audio, Laurence Chapman provides an orchestral soundtrack that evokes the epic feel of adventure films, particularly the Indiana Jones series. Musical stings play as new routes are unlocked. Sound effects accentuate the character of each locale: in Maghreb countries, we hear the noise of the souks; in rural regions, we hear crickets chirp; and at the North Pole, icy winds blow. The soundscape shifts as the players travel, with culture-specific instruments entering the ambience as Fogg and Passepartout visit cities in different regions of the world.

Spatiotemporally, the software is playable on mobile and desktop devices, which means that the games are portable; this also means that texts come in shorter bursts of reading so that play sessions can be terminated at any point. An average circumnavigation of the globe takes about two to three hours of solid reading, which is rather short for a game, but due to the branching narrative style, this brevity is ideal for repeated playthroughs. Even so, it is extremely unlikely that any player has read every piece of text the game has to offer.

Within the game, however, space and time are the main challenges. Aside from the titular eighty days in which to complete Fogg's journey, trains, ships and other transport options run at certain times of the day, and if Passepartout spends too much time buying and selling in the market, the player might miss an important connection. Time passes while travelling and also when exploring the cities. The only time when the clock is not ticking is when a large piece of text is displayed, which the player can read at their own pace to enjoy the quality of its writing. Also, Fogg must sleep comfortably during the night or risk compromising his health - and, as an ill Fogg cannot travel, it costs precious days to nurse him back to health.

As for the issue of space, the game encompasses all major continents with the exception of Antarctica, and the player is free to choose their route across the globe. In topological terms, the cities are laid out in a graph structure, with the cities as nodes and the trips as edges. Similar graphs could be drawn in other pieces of interactive fiction, which shows this title's connections to the genre. Likewise, many nineteenth- and twentieth-century board games using the theme of travel have used a graph-like structure to provide players with an opportunity to plan their journeys across the board. While a few routes are two-way, most journeys steadily push Fogg onwards, largely to the east, which further connects the gameplay to the family of board games called race games, and it fits thematically with Fogg's wager. Cities are more densely packed in Europe and South East Asia, while the longest journeys are the voyages crossing the Atlantic and the Pacific. Sometimes disaster strikes during a journey, and detours or route changes are a significant part of the game's challenge. In this way, the game represents the original novel's unforeseen circumstances, 


\section{6}

Péter Kristóf Makai

even though they happen in a deterministic fashion, as the end result of conditions tracked by the game.

A semiotic reading of the game may note that ostensibly the game takes place in a world that has the same geography as our world, represented on a stylized globe (an iconic relationship), with the same city names as on our planet referring to the game locations (a symbolic relationship), and when Fogg and Passepartout visit a city they see images of famous landmarks, drawn from photographs to match the art style of the game (an indexical relationship). Even so, reader-players will soon realize that this world is not exactly the same as Earth was in 1872: it has diverged from our history in important social, political and technological ways.

For example, several vehicles used for travel have no equivalent in our world: the moving city of Agra takes a real-world referent, the Indian city of Agra, but uses the genre conventions of steampunk fiction to reinvent it as a pneumatically moving city (a mixture of symbolic codes from fact and fiction). Similarly, submersible trains and mechanical palanquins are more or less plausible inventions that have no real-world referents, but they evoke the atmosphere of an alternative 1872, where the horizons of the future seemed limitless. Meanwhile, other modes of transport, such as the Bożek car or the Trevithick steam carriage, are inventions that have actual, albeit obscure references in the real world and appear faithfully (an indexical relationship): the Czech-Polish Józef Bożek and the Englishman Richard Trevithick, respectively, did create steam-powered vehicles, even if they are relegated to the annals of history.

Similarly, as an alternative history, the game's storyworld is a combination of accurate geopolitical entities and events on the one hand and clearly fictional additions on the other. For example, in the British, Ottoman and Chinese Empires, Prussia and France are present as world powers, but in this world, Prussia has invaded and occupied a significant portion of France with a superior force of automaton soldiers. Paris has held many world expositions during the relevant era, but the one Fogg visits in 1872 can only be fictional. The most crucial change to our world stems from the authors' anti-colonial world-building philosophy: the non-Euro-Atlantic world is a much more formidable, dominant force in the alternative history when compared to the allohistorical timeline. The resulting storyworld showcases a nuanced, intricate webwork of different empires, oppressive regimes, republics, small communes or resistance movements, most of which resent the European colonial powers.

\section{Summary: 80 days}

As this short analysis demonstrates, the design team of 80 Days used a variety of media to construct the storyworld that is meant to take the player on a fantastical journey inspired by Verne's globetrotting adventures. The visuals, sounds and writing liberally borrow from the literary genre of steampunk science 
fiction to reinforce a sense of living in an alternative 1872, where history plays out differently from events in our world. This supports the artistic philosophy of the team, which reimagines the political landscape of Earth from an anticolonial perspective. The alternative setting also helps the team to tell many different, sometimes mutually exclusive stories about Fogg and Passepartout's adventures, which illustrate, on a different level, that the course of history is not set in stone. All artistic choices contribute to a central vision of how the small decisions of individual humans can make a difference in the world, a theme that is explored in many computer games.

\section{References}

Aarseth, E.J. 1997. Cybertext perspectives on ergodic literature. Baltimore: Johns Hopkins University Press.

Barton, Matt and Stacks, Shane. 2019. Dungeons and desktops: The history of computer roleplaying games 2e. Boca Raton: A K Press/CRC Press. https://www.routledge.com/ Dungeons-and-Desktops-The-History-of-Computer-Role-Playing-Games-2e/Ba rton-Stacks/p/book/9781138574649

Black Mirror: Bandersnatch. 2018. Directed by David Slade. Written by Charlie Brooker. Netflix.

Cooper, J. 2019. Game anim: Video game animation Explained. Boca Raton: CRC Press. Ensslin, A. 2007. Canonizing hypertext: Explorations and constructions. New York: Continuum. Hayles, N.K. 2008. Electronic literature: New horizons for the literary. Notre Dame: University of Notre Dame.

Jerz, D.G. 2007. Somewhere nearby is a colossal cave: Examining Will Crowther's original Adventure in code and in Kentucky. Digital Humanities Quarterly, 1(2). http://digitalhuma nities.org//dhq/vol/1/2/000009/000009.html [Accessed 3 December 2020].

Kocurek, Carly A. 2015. Coin-operated Americans: Rebooting boyhood at the video game arcade. Minneapolis: University of Minnesota Press.

Montfort, N. 2003. Twisty little passages: An approach to interactive fiction. New York: MIT Press.

The Matrix. 1999. Directed by Lana Wachowski and Lily Wachowski. USA: Warner Bros. Pictures.

VanderMeer, A. and VanderMeer, J. 2008. Steampunk. San Francisco: Tachyon Publications.

Verne, J. 1864. Voyage au centre de la terre. Paris: Pierre-Jules Hetzel.

Verne, J. 1865. De la terre à la lune. Paris: Pierre-Jules Hetzel.

Verne, J. 1869-70. Vingt mille lieues sous les mers. Paris: Pierre-Jules Hetzel.

Verne, J. 1872. Le tour du monde en quatre-vingts jours. Paris: Pierre-Jules Hetzel.

\section{Games referenced}

80 Days. 2014. iOS and PC. Inkle Studios.

Adventure. 1975-6. PDP-10. Will Crowther and Don Woods.

Alan Wake. 2010. PC. Remedy Entertainment.

Another World. 1991. Amiga, Atari. Delphine Software, Interplay and The Removers.

Lunar Lander. 1979. Arcade. Atarti. Atarti Inc. 


\section{Péter Kristóf Makai}

Phantasmagoria. 1995. PC, Sega Saturn. Sierra On-Line.

Scribblenauts Unlimited. 2012. PC. 5th Cell.

The Neverhood. 1995. PC, PlayStation. The Neverhood, Inc. and Riverhillsoft.

The Typing of the Dead: Overkill. 2013. Wii, PlayStation3, PC, iOS, Android. Headstrong Games.

Toonstruck. 1996. PC. Burst Studios.

Type: Rider. 2013. PC, PlayStation 4, iOS. Agat Films \& Cie - Ex Nihilo.

Undertale. 2015. PC, OSX, Linux, PlayStation, Nintendo Switch. Toby Fox.

Wing Commander III: Heart of the Tiger. 1993. MS-DOS, macOS, PlayStation. Origin Systems.

World of Warcraft. 2004. Windows, macOS. Blizzard Entertainment 